\title{
SYMPLECTIC INTEGRATION OF CONSTRAINED HAMILTONIAN SYSTEMS
}

\author{
B. LEIMKUHLER AND S. REICH
}

\begin{abstract}
A Hamiltonian system in potential form $\left(H(q, p)=p^{t} M^{-1} p / 2+\right.$ $F(q)$ ) subject to smooth constraints on $q$ can be viewed as a Hamiltonian system on a manifold, but numerical computations must be performed in $\mathbf{R}^{n}$. In this paper, methods which reduce "Hamiltonian differential-algebraic equations" to ODEs in Euclidean space are examined. The authors study the construction of canonical parametrizations or local charts as well as methods based on the construction of ODE systems in the space in which the constraint manifold is embedded which preserve the constraint manifold as an invariant manifold. In each case, a Hamiltonian system of ordinary differential equations is produced. The stability of the constraint-invariants and the behavior of the original Hamiltonian along solutions are investigated both numerically and analytically.
\end{abstract}

\section{INTRODUCTION}

Consider a Hamiltonian system of the form

$$
\begin{gathered}
\dot{q}=M^{-1} p, \\
\dot{p}=-\nabla F(q),
\end{gathered}
$$

where $q, p \in \mathbf{R}^{n}, F: \mathbf{R}^{n} \rightarrow \mathbf{R}$ is $C^{2}$, and $M$ is a symmetric, positive definite $n \times n$ mass matrix. With the scaling $q \mapsto M^{1 / 2} q, p \mapsto M^{1 / 2} p$ we can reduce (1)-(2) to an equivalent system with $M=I$, so we will always assume this simplification in the remainder of the paper. All of the essential results of this paper could be extended to the separable case $(H(q, p)=T(p)+F(q))$. The system (1)-(2) arises in numerous practical applications (e.g., molecular dynamics [12]). The flow of a Hamiltonian system like (1)-(2) is symplectic, meaning that it conserves the two-form $d q \wedge d p .^{1}$ A growing body of numerical evidence suggests that the integration of (1)-(2) over long time intervals is best performed by canonical discretization schemes [20] which maintain the symplectic structure of the flow.

A natural question is what happens when (1)-(2) is constrained by algebraic equations in $q$ and/or $p$. In this paper, we primarily restrict ourselves to the

Received by the editor September 25, 1992 and, in revised form, September 2, 1993.

1991 Mathematics Subject Classification. Primary 65L05.

Key words and phrases. Differential-algebraic equations, constrained Hamiltonian systems, canonical discretization schemes, symplectic methods.

${ }^{1} d q \wedge d p=\sum_{i} d q_{i} \wedge d p_{i}$. The wedge product $\wedge$ is a bilinear and skew-symmetric form [2]. 


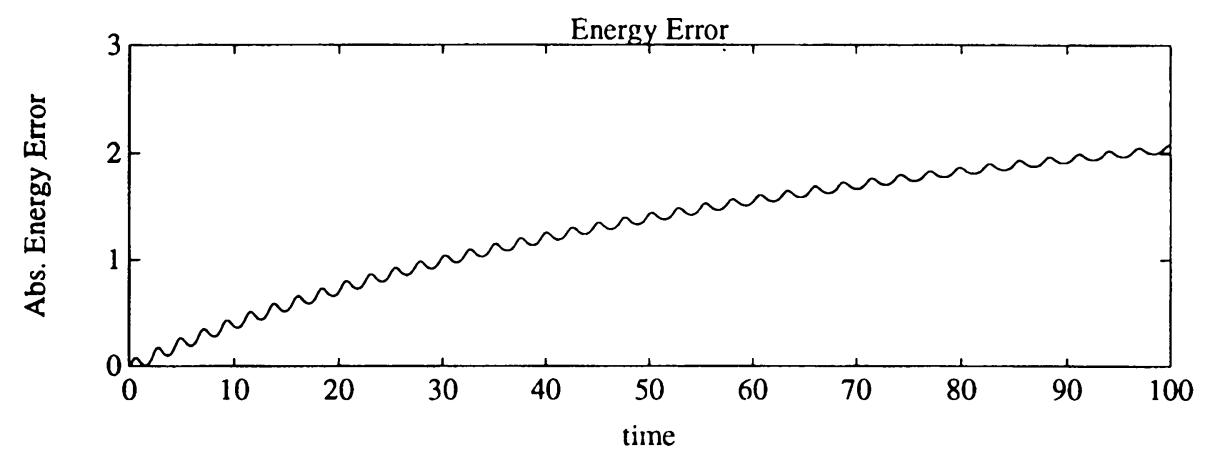

FIGURE 1. Energy error in BDF-2 solution vs. time

case when the constraints are holonomic (i.e., essentially dependent on $q$ only) as in many mechanical systems, in which case, starting from a Lagrangian variational principle, one would arrive at a system of differential-algebraic equations of the form

$$
\begin{gathered}
\dot{q}=p, \\
\dot{p}=-\nabla F(q)-G(q)^{t} \lambda, \\
0=g(q),
\end{gathered}
$$

where $g: \mathbf{R}^{n} \rightarrow \mathbf{R}^{m}, G(q)=g^{\prime}(q) \in \mathbf{R}^{m \times n}$ has full rank, and we have taken $M=I$. Furthermore, $\lambda \in \mathbf{R}^{m}$ is a vector of Lagrange multipliers. This system generates a flow on the $(2 n-2 m)$-dimensional manifold $\mathscr{M}=\{(q, p): g(q)=$ $0, G p=0\}$. (For notational simplicity, we write $G$ for $G(q)$, etc.)

A standard (nonsymplectic) approach to solving the constrained system (3)(5) is based on direct discretization with backward differentiation formulas (BDF methods) [5]. In Figure 1, we have indicated the typical growth in energy error in the solution of a simple plane pendulum (12)-(14) computed with the second-order BDF method (fixed stepsize $h=.05$ ). Here, the initial energy was $E(0)=.5$, so we have completely lost the conservative character of the problem after only a small number of periods.

Other approaches to solving (3)-(5) are based on the construction of various families of ODEs in Euclidean space: the underlying and state-space form ODEs. An example of an underlying ODE is obtained by first differentiating the constraint $g(q)=0$ and using (3):

$$
G \dot{q}=0=G p .
$$

Then differentiating again yields

$$
G \dot{p}+G_{q}(p, p)=0 .
$$

(We use the notation $G_{q}(p, w)$ to denote the derivative of $G$-the tensor second derivative of $g$-operating on vectors $p$ and $w$.) Next we substitute (4) and solve the resulting equations for $\lambda$ in terms of $q$ and $p$ :

$$
\lambda=\Lambda(q, p)=\left(G G^{t}\right)^{-1}\left(-G \nabla F(q)+G_{q}(p, p)\right),
$$


which, upon reintroduction in (4), gives

$$
\dot{p}=-(I-\mathscr{H}) \nabla F(q)-G^{t}\left(G G^{t}\right)^{-1} G_{q}(p, p),
$$

where $\mathscr{H}=G^{t}\left(G G^{t}\right)^{-1} G$ is the orthogonal projector onto the orthogonal complement of the null space of $G$. We term the ODE system comprising $\dot{q}=p$ together with (6) the standard underlying $O D E$; it has the feature that the flow it generates reduces to the flow of (3)-(5) along the constraint manifold $\mathscr{M}$. On the other hand, without enforcing the constraint, $(3),(6)$ actually define a flow in $\mathbf{R}^{2 n}$. Numerical methods applied directly to this underlying ODE typically drift from the constraint manifold into $\mathbf{R}^{2 n}$ during the course of integration, but a popular approach to short-time-interval computations incorporates numerical discretization of $(3),(6)$ and frequent projection onto the constraints $[9,3]$.

While (3), (6) define a particular underlying ODE, there is an entire family of ODEs whose dynamics reduce to those of the constrained system along $\mathscr{M}$. While (3), (6) is not a Hamiltonian system away from $\mathscr{M}$, Hamiltonian ODE systems can be found in the family of underlying ODEs; such systems are developed in $\S 3$, using the Poisson bracket formalism of Dirac [8] for constrained Hamiltonian systems.

The second family of ODEs associated with the DAE (3)-(5) is constructed via a parametrization of the constraint (5). Suppose there is a function $\phi: \mathbf{R}^{n-m}$ $\rightarrow \mathbf{R}^{n}$ with a full rank Jacobian satisfying, for all $\delta \in \mathbf{R}^{n-m}$,

$$
g(\phi(\delta))=0
$$

then, with $\delta, \theta \in \mathbf{R}^{n-m}$, the equations

$$
q=\phi(\delta), \quad p=\phi^{\prime}(\delta) \theta
$$

define an invertible map from $\mathscr{M}$ to $\mathbf{R}^{2 n-2 m}$. This results in equations in the new variables of the form

$$
\begin{aligned}
\phi^{\prime}(\delta) \dot{\delta} & =\phi^{\prime}(\delta) \theta \\
\phi^{\prime}(\delta) \dot{\theta}+\frac{\partial \phi^{\prime}(\delta) \theta}{\partial \delta} \dot{\delta} & =-\nabla F(\phi(\delta))+G^{t} \lambda
\end{aligned}
$$

Now multiplying both equations on the left by $\left(\phi^{\prime t} \phi^{\prime}\right)^{-1} \phi^{\prime t}$ results in

$$
\dot{\delta}=\theta, \quad \dot{\theta}=-\left(\phi^{\prime t} \phi^{\prime}\right)^{-1} \phi^{\prime t}\left(\nabla F(\phi(\delta))-\phi^{\prime \prime}(\theta, \theta)\right) .
$$

A state space form constructed along these lines will rarely be Hamiltonian. On the other hand, by searching among all parametrizations of $\mathscr{M}$ (which do not necessarily maintain the relation $\dot{\delta}=\theta$ ), one can find a family of canonical state space forms for the constrained problem. This is the approach taken in $\S 2$.

An alternative approach would be based on direct canonical discretization of the constrained system (see Leimkuhler and Skeel [12], Reich [18], and Jay [10]).

\section{HAMILTONIAN STATE SPACE FORMS}

The following theorem shows that there is a family of canonical state space forms based on parametrizations of the constraints. Throughout this section, we are concerned with a Hamiltonian of the form $H=F(q)+p^{t} p / 2$. 
Proposition 2.1. If $\phi$ is a local parametrization of $g(q)=0$, then the equations

$$
q=\phi(\delta), \quad \phi^{\prime t} p=\theta, \quad G p=0
$$

define a canonical map between $\mathscr{M}$ and an open subset of $\mathbf{R}^{2 n-2 m}$. The Hamiltonian in the new coordinates is

$$
\widehat{H}(\delta, \theta)=\frac{1}{2} \theta^{t}\left(\phi^{\prime t} \phi^{\prime}\right)^{-1} \theta+(F \circ \phi)(\delta) .
$$

Proof. To see that the mapping is canonical, note that

$$
d q \wedge d p=\left(\phi^{\prime}(\delta) d \delta\right) \wedge d p=d \delta \wedge\left(\phi^{\prime}(\delta)^{t} d p\right) ;
$$

now $d \theta=\phi^{\prime}(\delta)^{t} d p+\sum_{i=1}^{n} p_{i} \phi_{i}^{\prime \prime} d \delta$, where $\phi^{\prime \prime}$ represents the Hessian of the $i$ th component of $g$, so

$$
\begin{aligned}
d \delta \wedge\left(\phi^{\prime}(\delta)^{t} d p\right) & =d \delta \wedge\left(d \theta-\sum_{i=1}^{n} p_{i} \phi_{i}^{\prime \prime} d \delta\right) \\
& =d \delta \wedge d \theta-\sum_{i=1}^{n} p_{i} d \delta \wedge \phi_{i}^{\prime \prime} d \delta
\end{aligned}
$$

Using the properties of the wedge product, we can show in a straightforward way that $d u \wedge B d u$ vanishes when $B$ is a symmetric matrix; thus we conclude

$$
d q \wedge d p=d \delta \wedge d \theta .
$$

It is easy to see that the potential energy in the new coordinates becomes $(F \circ \phi)(\delta)$; to derive an expression for the kinetic energy in the new coordinates, observe that

$$
p=\left[\begin{array}{c}
\phi^{\prime t} \\
g^{\prime}(q)
\end{array}\right]^{-1}\left[\begin{array}{l}
\theta \\
0
\end{array}\right]
$$

and hence

$$
\frac{1}{2} p^{t} p=\frac{1}{2}\left[\begin{array}{ll}
\theta^{t} & 0]
\end{array}\left[\begin{array}{l}
\phi^{\prime t} \\
g^{\prime}
\end{array}\right]^{-t}\left[\begin{array}{l}
\phi^{\prime t} \\
g^{\prime}
\end{array}\right]^{-1}\left[\begin{array}{l}
\theta \\
0
\end{array}\right]=\frac{1}{2}\left[\begin{array}{ll}
\theta^{t} & 0]
\end{array}\left[\begin{array}{cc}
\phi^{\prime t} \phi^{\prime} & \phi^{\prime t} g^{\prime t} \\
g^{\prime} \phi^{\prime} & g^{\prime} g^{\prime t}
\end{array}\right]^{-1}\left[\begin{array}{l}
\theta \\
0
\end{array}\right] .\right.\right.
$$

But we know that $g^{\prime} \phi^{\prime}=0$ since $\phi$ is a parametrization of $g$; hence we have

$$
\frac{1}{2} p^{t} p=\frac{1}{2} \theta^{t}\left(\phi^{\prime t} \phi^{\prime}\right)^{-1} \theta
$$

and the result follows.

In general, such a state space form is computationally impractical because of the need to automatically obtain, and then twice differentiate, the function $\phi$ defining the parametrization. In certain cases, for example a many-body system whose constraints have a tree structure, one can derive a global parametrization of the Hamiltonian directly from physical principles. For the general case, we show how a careful choice of parametrization can lead to a more general formulation without explicit knowledge of $\widehat{H}$.

Following Potra and Rheinboldt [17], we define $q=\phi(\delta)$ as the solution of the nonlinear system

$$
A q=\delta,
$$

$$
g(q)=0,
$$


where the constant matrix $A \in \mathbf{R}^{(n-m) \times n}$ is chosen so that $R=\left[\begin{array}{l}A \\ G\end{array}\right]$ is a nonsingular matrix. Typically, $A$ is treated as a piecewise constant function of time. Previous authors have used the induced state space form obtained by setting $\delta^{\prime}=\theta$ to solve multibody dynamics problems, but instead, we here choose $\theta$ according to Proposition 2.1 to insure a canonical map.

If the mapping $\phi=\phi(\delta)$ in Proposition 2.1 is defined by (7)-(8), then $\phi^{\prime}$ can be written explicitly as

$$
\phi^{\prime}=R^{-1}\left[\begin{array}{l}
I \\
0
\end{array}\right] .
$$

Hence, the equations determining $\theta$ boil down to

$$
\left[\begin{array}{ll}
I & 0
\end{array}\right] R^{-t} p=\theta, \quad G p=0 .
$$

Thus, we must have

$$
R^{-t} p=\left[\begin{array}{c}
\theta \\
\boldsymbol{\theta}
\end{array}\right]=: b
$$

for some $\boldsymbol{\theta}$; hence $p=R^{t} b=A^{t} \theta+G^{t} \Theta$. Now by virtue of $G p=0$, we obtain

$$
p=(I-\mathscr{H}) A^{t} \theta
$$

with $\mathscr{H}=G^{t}\left(G G^{t}\right)^{-1} G$.

Theorem 2.2. Suppose a parametrization of $g(q)=0$ is defined via (7)-(8). Then the corresponding Hamiltonian state space form is characterized by

$$
\begin{aligned}
\dot{\delta} & =A(I-\mathscr{H}) A^{t} \theta \\
A(I-\mathscr{H}) A^{t} \dot{\theta} & =-A(I-\mathscr{H}) \nabla F(q)+A(I-\mathscr{H}) \mathscr{H}_{q}\left(p, A^{t} \theta\right),
\end{aligned}
$$

since $A(I-\mathscr{H}) A^{t}$ is nonsingular.

Proof. Differentiating (7) with respect to time and using (9) yields

$$
\dot{\delta}=A \dot{q}=A p=A(I-\mathscr{H}) A^{t} \theta .
$$

Next differentiate (9) with respect to time, replace $\dot{p}$ by (6), and premultiply by $A(I-\mathscr{H})$ to obtain equation (11).

It must be pointed out that although we began this section treating a problem with a separable Hamiltonian (i.e., $H(q, p)=T(p)+V(q))$, the Hamiltonian of the canonical state space form ODE is not separable. Since no explicit symplectic discretizations are available for a general Hamiltonian, it would be necessary to employ an implicit scheme. In [11], it is shown that the mixed set of equations (7)-(11) in $q, p, \delta$, and $\theta$ can be solved effectively with Gauss-Legendre Runge-Kutta discretization by an algorithm based on functional iteration. However, there is a more serious and perhaps insurmountable problem with using the discretized state space form for symplectic integration.

Recent results (see, e.g., Sanz-Serna [20]) indicate that an integrator for a Hamiltonian system should consist of the iteration of one and the same symplectic map. In this case, it can be shown that there is a nearby Hamiltonian for which the numerical solution is nearly the exact flow. In terms of our state space form this means that the matrix $A$ must be held constant; in other words, $A$ must define a parametrization valid along the entire trajectory. 


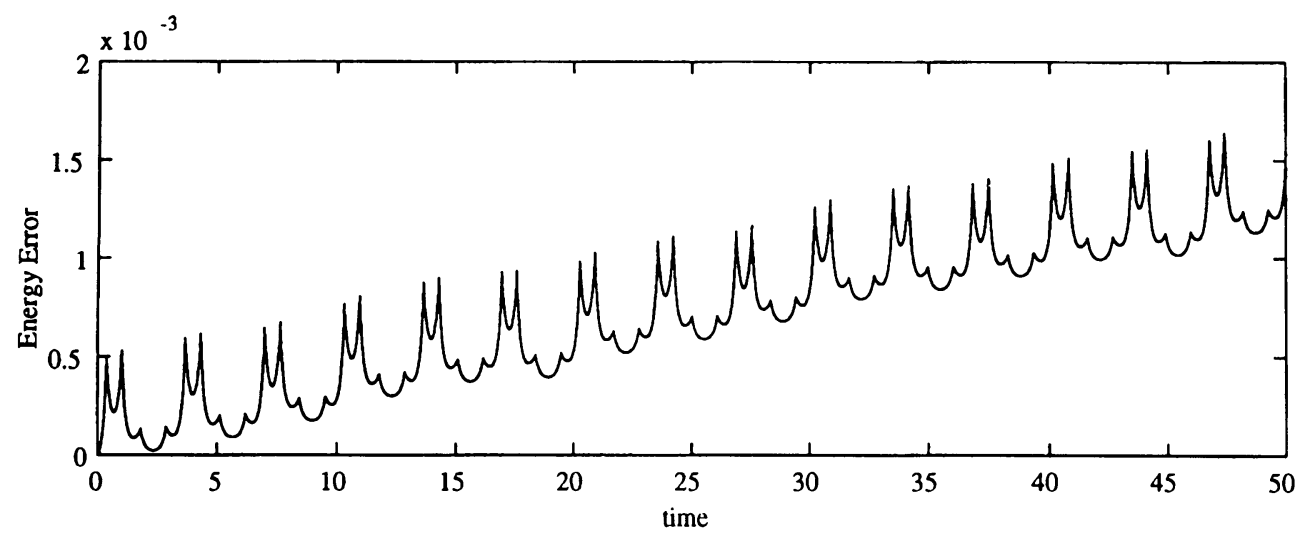

FIGURE 2. Energy error in numerically computed state space form vs. time

To illustrate the difficulty when the parametrization changes along a trajectory (i.e., when we switch from one local chart of the manifold to another), we consider the plane pendulum with unit length and mass, where for $q, p \in \mathbf{R}^{2}$, we have

$$
\begin{gathered}
H(q, p)=\frac{1}{2} p^{t} p+g q_{2}, \\
\dot{q}=p, \\
\dot{p}=\left[\begin{array}{c}
0 \\
-g
\end{array}\right]-\lambda q, \\
0=\frac{1}{2}\left(q^{t} q-1\right) .
\end{gathered}
$$

We parametrized the unit circle in four charts, $\Omega_{i}, i=1, \ldots, 4$, using alternately $x$ and $y$ as parameter, and following the program of Theorem 2.2. The chart was changed when $y$ crossed the threshold values $\pm \sqrt{2} / 2$. For our experiment, we took $g=0$ and set $\left(q_{1}(0), q_{2}(0), p_{1}(0), p_{2}(0)\right)=$ $(1,0,0,-2)$. In each chart, we applied the implicit midpoint method. This resulted in correct dynamics on bounded intervals as $h \rightarrow 0$.

As illustrated in Figure 2 (with $h=.01$ ), we observed an undesirable drift in the energy of the numerical solution. Such behavior would not be anticipated from fixed stepsize symplectic integration of a single Hamiltonian vector field. Nevertheless, the numerical results for the Hamiltonian state space form were a vast improvement over the results with BDF- 2 .

\section{HAMILTONIAN UNDERLYING ODES}

We now examine the possibility of obtaining Hamiltonian underlying ODEs as an alternative to the computation of the state space form. In case the constraint is linear, $G q=0$, with $G$ constant, the standard underlying ODE (1)(2) reduces to

$$
\dot{q}=p, \quad \dot{p}=-(I-\mathscr{H}) \nabla F(q) .
$$

This ODE system is not Hamiltonian because the projection of $\nabla F$ is not necessarily the gradient of any function; however, it is easy to construct an 
underlying ODE which is Hamiltonian: we simply note that if $q$ lies on $G q=$ 0 , then $(I-\mathscr{H}) q=q$, so that

$$
\dot{q}=p, \quad \dot{p}=-(I-\mathscr{H}) \nabla F((I-\mathscr{H}) q)
$$

is also an underlying ODE-and this one is a Hamiltonian system.

For the nonlinearly constrained case, we make use of Dirac's theory of constrained Hamiltonian systems [8].

3.1. Nonlinearly constrained Hamiltonians. In this subsection, we will derive a modified, unconstrained Hamiltonian with the property that on $\mathscr{M}$ the modified and the original Hamiltonian are identical and that $\mathscr{M}$ is an invariant manifold of the flow corresponding to the modified Hamiltonian. As a result, we will obtain a Hamiltonian ODE whose flow on $\mathscr{M}$ reduces to the flow of (3)-(5). The main idea in the construction of the modified Hamiltonian is the following: for a Hamiltonian function $H=H(q, p)$ and a scalar-valued function $\phi=\phi(q, p)$, the condition for $\phi$ to be an invariant under the flow of the Hamiltonian system derived from $H$ is just that the Poisson bracket [17] of $\phi$ with $H$ vanishes, i.e.,

$$
\sum_{i=1}^{n} \frac{\partial \phi}{\partial q_{i}} \frac{\partial H}{\partial p_{i}}-\frac{\partial \phi}{\partial p_{i}} \frac{\partial H}{\partial q_{i}}=:\{\phi, H\}=0
$$

Following Dirac [8], we make a distinction between two types of invariants. $\phi(q, p)=0$ is said to be a strong invariant of the flow derived from $H$ in case $\{\phi, H\}$ vanishes identically. A weak invariant is one that satisfies $\{\phi, H\}=0$ only when $\phi(q, p)=0$. In the latter case we will often write $\{\phi, H\} \approx 0$. We make use of the following elementary properties of Poisson brackets for functions $\phi, \psi, \omega: \mathbf{R}^{2 n} \rightarrow \mathbf{R}$ and real constants $\alpha_{1}, \alpha_{2}$ :

(i) $\{\phi, \psi\}=-\{\psi, \phi\}$,

(ii) $\{\phi, \phi\}=0$,

(iii) $\left\{\alpha_{1} \phi_{1}+\alpha_{2} \phi_{2}, \psi\right\}=\alpha_{1}\left\{\phi_{1}, \psi\right\}+\alpha_{2}\left\{\phi_{2}, \psi\right\}$; $\left\{\psi, \alpha_{1} \phi_{1}+\alpha_{2} \phi_{2}\right\}=\alpha_{1}\left\{\psi, \phi_{1}\right\}+\alpha_{2}\left\{\psi, \phi_{2}\right\}$

(iv) $\{\phi, \psi \omega\}=\{\phi, \psi\} \omega+\{\phi, \omega\} \psi$.

If $\phi$ is not an invariant of the Hamiltonian $H$, as in the case of constrained Hamiltonian systems with $H=p^{t} p / 2+F(q)$ and $\phi=g$, consider the adjusted (constrained) Hamiltonian function

$$
H_{T}^{(1)}=H+\mu \phi
$$

Here the function $\mu=\mu(q, p)$ plays much the same role as the Lagrange multiplier in (3)-(5). The function $\mu$ is chosen to insure $\phi=0$ along solutions, which certainly holds if $\phi$ is a weak invariant of the flow of $H_{T}^{(1)}$. For this to happen, we need that

$$
0 \approx\left\{\phi, H_{T}^{(1)}\right\}=\{\phi, H\}+\{\phi, \mu \phi\}=\{\phi, H\}+\{\phi, \phi\} \mu+\{\phi, \mu\} \phi
$$

Taking $\phi=0$ in the above, and noting that $\{\phi, \phi\}=0$, we must have $\{\phi, H\} \approx 0$. Since we assumed $\{\phi, H\} \neq 0$, we have to treat the equation $\psi \equiv\{\phi, H\}=0$ as a new constraint and consider the revised Hamiltonian

$$
H_{T}^{(2)}=H+\mu_{1} \phi+\mu_{2} \psi
$$


If we now seek $\mu_{1}=\mu_{1}(q, p)$ and $\mu_{2}=\mu_{2}(q, p)$ to insure that both $\left\{\phi, H_{T}^{(2)}\right\}$ $\approx 0$ and $\left\{\psi, H_{T}^{(2)}\right\} \approx 0$, we find that the key issue concerns the invertibility of the matrix of Poisson brackets,

$$
R=\left[\begin{array}{cc}
\{\phi, \phi\} & \{\phi, \psi\} \\
\{\psi, \phi\} & \{\psi, \psi\}
\end{array}\right]=\left[\begin{array}{cc}
0 & \{\phi, \psi\} \\
\{\psi, \phi\} & 0
\end{array}\right] .
$$

When $\{\phi, \psi\} \neq 0$, then $R$ is nonsingular, and we can solve for the functions $\left(\mu_{1}, \mu_{2}\right)$ so that both $\phi=0$ and $\psi=0$ are invariant for $H_{T}^{(2)}$. Furthermore, on $\phi=\psi=0$ we have $H_{T}^{(2)}=H$.

We now turn to the case of a vector-valued constraint function. The main thing to bear in mind here is that, in the end, the constraints must be treated all at once, not one at a time. Given a vector of constraints $\phi=0$, one must first augment these constraints by all of the "hidden" constraints which arise by taking Poisson brackets with the augmented Hamiltonians, i.e., through the recursive differentiation of the constraints and substitution of the differential equations derived from the Hamiltonian. This approach is taken in [13] in deriving control laws for constrained systems, where it is shown that two steps of the reduction process are sufficient if the constraints are independent and holonomic, i.e., essentially only dependent on $q$.

As an example, if we follow the reduction for $H=p^{t} p / 2+F(q)$ and independent constraints of the form $g(q)=0$, we obtain the hidden constraints $G(q) p=0$. The next step is the construction of the modified Hamiltonian $H_{T}$ from $H$ and the constraints; thus we set

$$
H_{T}(q, p):=H(q, p)+\mu^{t} g(q)+\eta^{t} G(q) p .
$$

Equations for $\mu$ and $\eta$ can be derived directly by insuring that $g(q)=0$ and $G(q) p=0$ are either weak or strong invariants of the flow derived from $H_{T}$. A slight generalization of the Poisson bracket notation to handle multiple constraints makes this straightforward.

Definiton 3.1. Given vector-valued functions $\phi: \mathbf{R}^{2 n} \rightarrow \mathbf{R}^{l}$ and $\psi: \mathbf{R}^{2 n} \rightarrow \mathbf{R}^{m}$, the Poisson bracket of $\phi$ and $\psi$ is the $l \times m$ matrix whose $(i, j)$-component is defined by

$$
(\{\phi, \psi\})_{i, j}=\left\{\phi_{i}, \psi_{j}\right\} .
$$

The following proposition shows how the generalized Poisson bracket can be evaluated in terms of the Jacobians of the vector functions.

Proposition 3.1. Given vector-valued functions $\phi: \mathbf{R}^{2 n} \rightarrow \mathbf{R}^{l}$ and $\psi: \mathbf{R}^{2 n} \rightarrow \mathbf{R}^{m}$, let $\phi_{q}, \phi_{p} \in \mathbf{R}^{l \times n}, \psi_{q}, \psi_{p} \in \mathbf{R}^{m \times n}$, and denote the Jacobian matrices of the indicated function with respect to the indicated variables. Then

$$
\{\phi, \psi\}=\phi_{q} \psi_{p}^{t}-\phi_{p} \psi_{q}^{t}
$$

Using Proposition 3.1, we can easily see that $\{\phi, \psi\}=-\{\psi, \phi\}^{t}$. Proposition 3.2 is also useful in calculations:

Proposition 3.2. If $\phi$ and $\psi$ are as in Proposition 3.1, and $\lambda: \mathbf{R}^{2 n} \rightarrow \mathbf{R}^{m}$, then

$$
\left\{\phi, \lambda^{t} \psi\right\}=\{\phi, \psi\} \lambda+\{\phi, \lambda\} \psi .
$$


Proof. We have

$$
\begin{aligned}
\left\{\phi, \lambda^{t} \psi\right\} & =\phi_{q}\left(\lambda^{t} \psi\right)_{p}^{t}-\phi_{p}\left(\lambda^{t} \psi\right)_{q}^{t} \\
& =\phi_{q}\left(\psi_{p}^{t} \lambda+\psi^{t} \lambda_{p}\right)-\phi_{p}\left(\psi_{q}^{t} \lambda+\psi^{t} \lambda_{q}\right) \\
& =\{\phi, \psi\} \lambda+\{\phi, \lambda\} \psi . \quad
\end{aligned}
$$

The generalized Poisson bracket described here is purely a computational device and not technically a Poisson bracket in the classical sense (see, e.g., [17]). In particular, the Poisson bracket of a vector function with itself is a skew-symmetric matrix.

To get an invariant, we require

$$
\begin{array}{r}
\left\{g, H_{T}\right\}=\{g, H\}+\left\{g, \mu^{t} g\right\}+\left\{g, \eta^{t} G p\right\}=0, \\
\left\{G p, H_{T}\right\}=\{G p, H\}+\left\{G p, \mu^{t} g\right\}+\left\{G p, \eta^{t} G p\right\}=0 .
\end{array}
$$

Working out the Poisson brackets in the first equation, we get

$$
-\{g, H\}=\{g, g\} \mu+\{g, \mu\} g+\{g, G p\} \eta+\{g, \eta\} G p .
$$

If we do not take the constraints to be satisfied and seek $\mu$ and $\eta$ so that, e.g., $\left\{g, H_{T}\right\} \equiv 0$, then we need to solve a system of partial differential equations which actually becomes singular along the constraints; thus it seems to be too much to ask for strong invariance of the constraints.

On the other hand, for a weak invariant, we may assume that $g=G p=0$. Next, note that $\{g, g\}$ vanishes because $g$ is a function of $q$ only. Moreover, $\{g, G p\}=G G^{t}$, and thus

$$
-\{g, H\}=G G^{t} \eta \text {. }
$$

This can be solved for $\eta$ provided $G$ has full rank.

The second equation can be reduced to

$$
-\{G p, H\}=-G G^{t} \mu+\{G p, \mu\} g+\left[(G p)_{q} G^{t}-G(G p)_{q}^{t}\right] \eta-\{G p, \eta\} G p .
$$

Again, for weak invariance, the terms multiplied by $g$ and $G p$ drop out and we are left with equations which uniquely determine $\mu$. Once $\mu$ and $\eta$ are known, the Hamiltonian function $H_{T}$ is determined and the unconstrained equations of motion can be found by differentiating $H_{T}$ :

$$
\begin{aligned}
& \dot{q}=p+\mu_{p}^{t} g+\eta_{p}^{t} G p+G^{t} \eta, \\
& \dot{p}=-\nabla F-\mu_{q}^{t} g-G^{t} \mu-\eta_{q}^{t} G p-(G p)_{q}^{t} \eta .
\end{aligned}
$$

From a computational point of view, it may be quite involved to formulate the system in this manner. In particular, we now need to compute third derivatives of $g$ and second derivatives of $F$. Below we will consider some simplifications in the hope of improving the computational efficacy of Hamiltonian formulation.

In Figure 3 (next page), a numerical experiment with the Hamiltonian underlying ODE for the nonlinear pendulum (12)-(14) in Cartesian coordinates is summarized. We computed $\mu$ and $\eta$ as described above. Starting from the initial configuration $\left(q_{1}, q_{2}, p_{1}, p_{2}\right)=(1,0,0,-2)$, we solved the resulting Hamiltonian underlying ODE (16)-(17), using the implicit midpoint method and $h=.1$. The upper graph in Figure 3 demonstrates that the Hamiltonian 

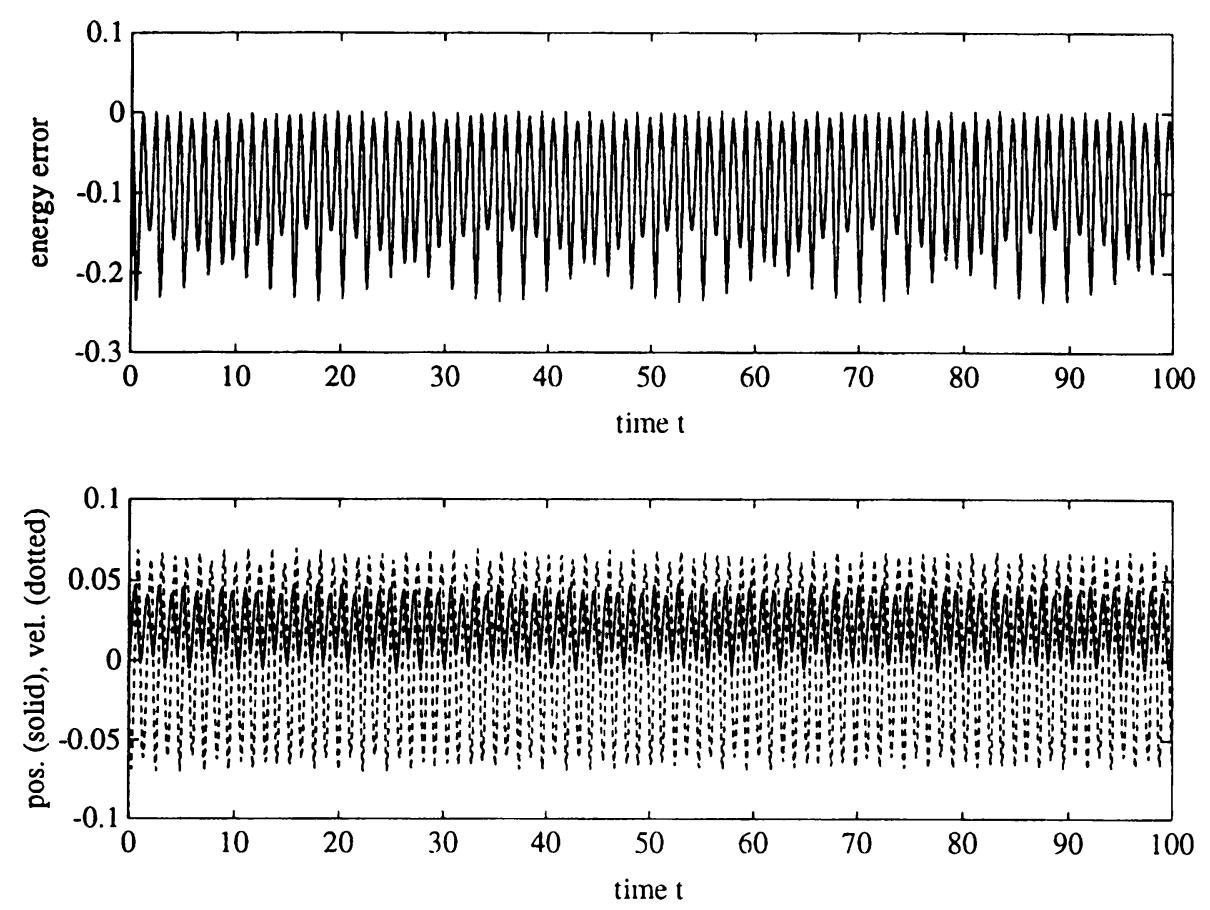

FIGURE 3. The Dirac underlying ODE for the pendulum; constraint residuals and Hamiltonian function

is approximately conserved over a relatively long time interval; the lower figure shows the extent to which the position and velocity constraint residuals are maintained during integration.

Figure 3 appears to contradict a result in Cooper [6] that says that quadratic invariants are exactly maintained by one-step methods (like the implicit midpoint method) which are "marginally algebraically stable". However, the invariants in [6] are always taken to be strong invariants (first integrals), which implies that the invariant manifold is in a certain sense locally stable; as we see below, this is not the case for a weak invariant.

3.2. A simplification. The process outlined above for obtaining a weak invariant is not completely well defined. For example, the determination of $\mu$ from

$$
-\{g, H\}=G G^{t} \eta
$$

can be done in any number of ways if we are allowed to freely use the relation $g=0$ or $G p=0$. For example, taking

$$
-\{g, H\}+\alpha g=G G^{t} \eta
$$

does not cause $g=0$ to cease to be an invariant of the flow ultimately obtained, but it may change characteristics of that flow for points near $\mathscr{M}$ where $g \neq$ 0 . We may also note that if $H(q, p)=F(q)+\frac{p^{t} p}{2}$ and $g=g(q)$, then $\{g, H\}=G p$, which is weakly zero when we are constraining with respect to both $g(q)=0$ and $G p=0$. This means that we have the obvious choice of taking $\{g, H\}=0$, which leads to $\eta=0$, or to follow the derivation as outlined above, which would lead to $\eta \neq 0$ away from $\mathscr{M}$. There is no obvious, a priori reason to favor one of these formulations over the other. 

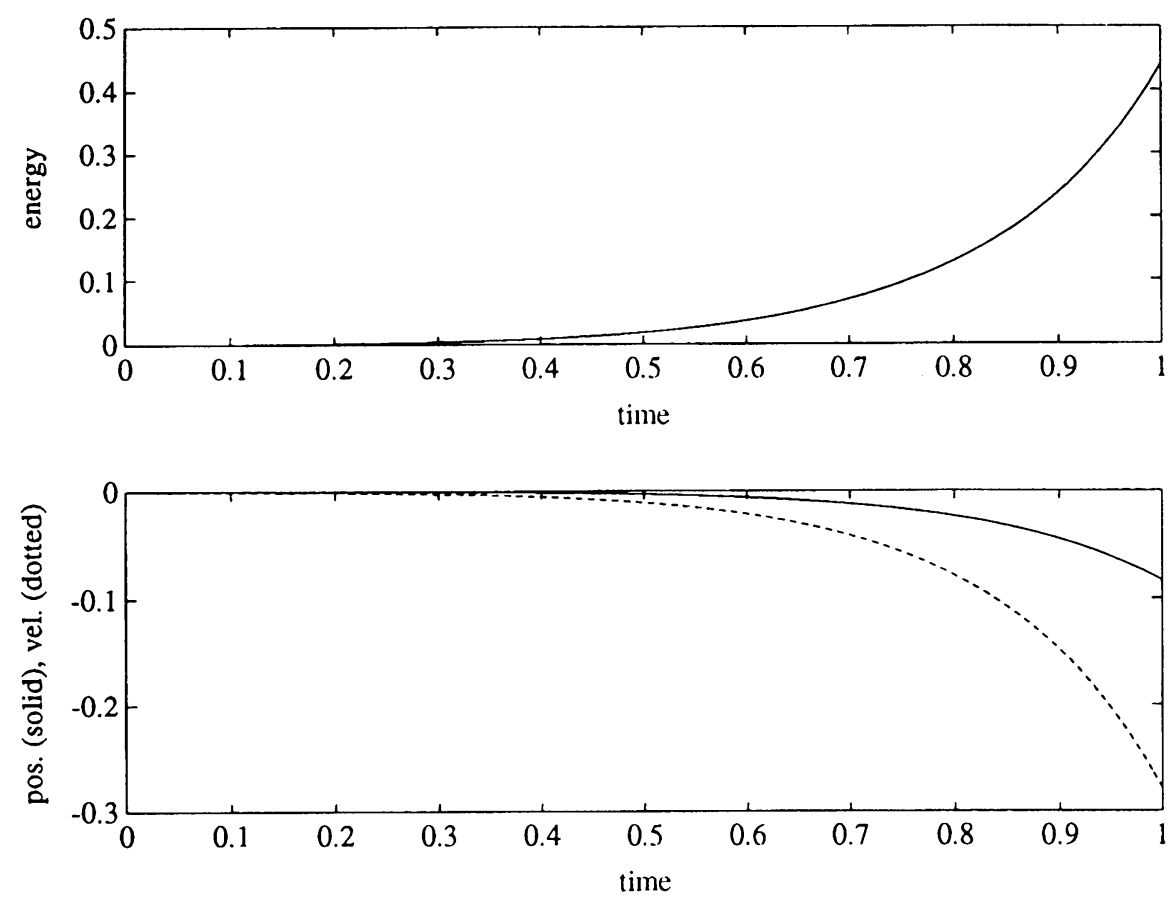

FIGURE 4. Hamiltonian function and constraint residuals for the simplified Hamiltonian formulation

If we take $\eta=0$, we get

$$
H_{T}=H+\mu^{t} g,
$$

so that, after insuring that $\mathscr{M}$ is invariant, we arrive at

$$
\begin{aligned}
& \dot{q}=p+\mu_{p}^{t} g, \\
& \dot{p}=-\nabla F-\mu_{q}^{t} g-G^{t} \mu,
\end{aligned}
$$

where $\mu=\left(G G^{t}\right)^{-1}\left(G \nabla F-G_{q}(p, p)\right)$. This system requires the computation of third derivatives of $g$ and second derivatives of $H$ as before.

Besides providing a simplified Hamiltonian formulation, (18)-(19) has the immediate and natural consequence of showing that along the constraint $(g=0)$, the standard underlying ODE generates a Hamiltonian flow. However, as shown in the next section, the formulation (18)-(19) can possess a somewhat surprising instability, which can be observed in computations whenever numerical discretization induces a perturbation of the constraint. In Figure 4, the implicit midpoint method (a canonical discretization scheme) has been applied to solve (18)-(19) for the Cartesian pendulum discussed above with fixed stepsize $h=.01$ from $t=0$ to $t=1$ with the same initial conditions as for Figure 3. Although the wedge product is maintained in this case, the constraint residuals and the Hamiltonian function are very rapidly growing in time.

3.3. Stability of the constraint-invariants. Let us begin with the case of a linearly constrained quadratic Hamiltonian with constraint $\phi=G q$ and $G$ 
constant. Here we find that the simplified Hamiltonian system based on $\widehat{H}_{T}=$ $H+\mu^{t} G q$ is

$$
\dot{q}=p, \quad \dot{p}=-(I-\mathscr{C}) \nabla F+D^{2} F \mathscr{H} q,
$$

where $D^{2} F$ is the Hessian matrix of $F$. Multiplying both equations by the (here constant) projector $\mathscr{H}$, we obtain

$$
\mathscr{H} \dot{q}=\mathscr{H} p, \quad \mathscr{H} \dot{p}=\mathscr{H} D^{2} F \mathscr{H} q .
$$

Since $\mathscr{H}=\mathscr{H}^{2}$, we can change to variables $r=\mathscr{H} q, s=\mathscr{H} p$ and write

$$
\begin{aligned}
& \dot{r}=s, \\
& \dot{s}=B r,
\end{aligned}
$$

where $B=\mathscr{H} D^{2} F \mathscr{H}$. Invariance of the constraints translates to $r=s=0$. If the Hessian is constant and positive definite, as is the case near a stable equilibrium, $B$ is positive semidefinite, and the equilibrium position in (20)(21) will be a saddle point. In this situation, one can expect an instability under perturbation of the constraint-invariants introduced via discretization.

If we perform a similar analysis starting from the Hamiltonian $H_{T}=\frac{p^{t} p}{2}+$ $F(q)+\mu^{t} G q+\eta^{t} G p$ of Dirac, we arrive at equations

$$
\dot{q}=(I-2 \mathscr{H}) p, \quad \dot{p}=-(I-\mathscr{H}) \nabla F+D^{2} F \mathscr{H} q .
$$

Multiplying the equations by $\mathscr{H}$, we get

$$
\mathscr{H} \dot{q}=\mathscr{H}(I-2 \mathscr{H}) p=-\mathscr{H} p, \quad \mathscr{H} \dot{p}=\mathscr{H} D^{2} F \mathscr{H} q .
$$

Hence, the corresponding system of differential equations for the constraint residuals is

$$
\begin{aligned}
& \dot{r}=-s, \\
& \dot{s}=B r .
\end{aligned}
$$

Now the equilibrium position $r=s=0$ has become a stable center under the assumption that $D^{2} F$ is positive definite.

3.4. Nonlinear constraints. We begin the discussion by writing the equations of motion for both formulations in the case of a single position constraint $\phi$. We derive a constraint of the form $\psi=\{\phi, H\}$. Assuming $\{\phi, \psi\} \neq 0$, we arrive at the Hamiltonian $H_{T}=H+\mu^{t} \phi+\eta^{t} \psi$. The conditions on $\mu$ and $\eta$ reduce to

$$
\begin{aligned}
\left\{\phi, H_{T}\right\} \approx 0 & \Rightarrow\{\phi, H\}+\{\phi, \psi\} \eta=0, \\
\left\{\psi, H_{T}\right\} \approx 0 & \Rightarrow\{\psi, H\}+\{\psi, \phi\} \mu+\{\psi, \psi\} \eta=0 .
\end{aligned}
$$

Since $\{\psi, \phi\}$ is an invertible matrix, and using $\{\phi, H\}=\psi$, we have

$$
\begin{aligned}
& \eta=-\{\phi, \psi\}^{-1} \psi, \\
& \mu=-\{\psi, \phi\}^{-1}\left[\{\psi, H\}-\{\psi, \psi\}\{\phi, \psi\}^{-1} \psi\right] .
\end{aligned}
$$


Now $\{\phi, \phi\}=0$. Also, because the constraint is scalar, $\{\psi, \psi\}=0$. Next, we write differential equations for the constraint residuals; thus

$$
\begin{aligned}
\dot{\phi}= & \left\{\phi, H_{t}\right\}=\psi+\left\{\phi,-\{\psi, \phi\}^{-1}\left[\{\psi, H\}=\{\psi, \psi\}\{\phi, \psi\}^{-1} \psi\right]\right\} \phi \\
& +\left\{\phi,-\{\phi, \psi\}^{-1} \psi\right\} \psi+\{\phi, \psi\}\left[-\{\phi, \psi\}^{-1} \psi\right], \\
\dot{\psi}=\{ & \left\{\psi, H_{T}\right\}=\{\psi, H\}+\left\{\psi,-\{\psi, \phi\}^{-1}\left[\{\psi, H\}-\{\psi, \psi\}\{\phi, \psi\}^{-1} \psi\right]\right\} \phi \\
& +\{\psi, \phi\}\left[-\{\psi, \phi\}^{-1}\left(\{\psi, H\}-\{\psi, \psi\}\{\phi, \psi\}^{-1} \psi\right)\right] \\
& +\left\{\psi,-\{\phi, \psi\}^{-1} \psi\right\} \psi+\{\psi, \psi\}\left[-\{\phi, \psi\}^{-1} \psi\right] .
\end{aligned}
$$

This simplifies to the system

$$
\begin{aligned}
\dot{\phi} & =-\left\{\phi,\{\psi, \phi\}^{-1}\{\psi, H\}\right\} \phi-\left\{\phi,\{\phi, \psi\}^{-1} \psi\right\} \psi, \\
\dot{\psi} & =-\left\{\psi,\{\psi, \phi\}^{-1}\{\psi, H\}\right\} \phi-\left\{\psi,\{\phi, \psi\}^{-1} \psi\right\} \psi .
\end{aligned}
$$

Now by employing the product rule for Poisson brackets (Proposition 3.2), we get

$$
\left\{\phi,\{\phi, \psi\}^{-1} \psi\right\} \psi=\left\{\phi,\{\phi, \psi\}^{-1}\right\} \psi^{2}+\{\phi, \psi\}\{\phi, \psi\}^{-1} \psi=\psi+O\left(\psi^{2}\right) .
$$

Similarly, we have

$$
\left\{\psi,\{\phi, \psi\}^{-1} \psi\right\} \psi=\left\{\psi,\{\phi, \psi\}^{-1}\right\} \psi^{2}+\{\psi, \psi\}\{\phi, \psi\}^{-1} \psi=O\left(\psi^{2}\right) .
$$

Thus, in the neighborhood of the constraint manifold, we have the following driving differential equations for the residuals:

$$
\begin{aligned}
\dot{\phi} & =-\psi-\left\{\phi,\{\psi, \phi\}^{-1}\{\psi, H\}\right\} \phi, \\
\dot{\psi} & =-\left\{\psi,\{\psi, \phi\}^{-1}\{\psi, H\}\right\} \phi .
\end{aligned}
$$

On the other hand, if we start with $\widehat{H}_{T}=H+\mu^{t} \phi$, then we obtain via the same sort of calculations

$$
\begin{aligned}
\dot{\phi} & =\psi-\left\{\phi,\{\psi, \phi\}^{-1}\{\psi, H\}\right\} \phi, \\
\dot{\psi} & =-\left\{\psi,\{\psi, \phi\}^{-1}\{\psi, H\}\right\} \phi .
\end{aligned}
$$

Note that the only difference between (26)-(27) and (28)-(29) is the sign that appears with $\psi$ in the first equation of each system.

Let us turn to an example. For the Cartesian pendulum with zero gravity, $\phi=\left(q^{t} q-1\right) / 2$ and $\psi=q^{t} p$. The Dirac Hamiltonian becomes

$$
H_{T}(q, p)=\frac{p^{t} p}{2}+\frac{1}{2} \frac{p^{t} p}{q^{t} q}\left(q^{t} q-1\right)-\frac{\left(q^{t} p\right)^{2}}{q^{t} q}
$$

and the corresponding ODE system is

$$
\begin{aligned}
& \dot{q}=\left(\frac{2 q^{t} q-1}{q^{t} q} I-\frac{2}{q^{t} q} q q^{t}\right) p \\
& \dot{p}=-\frac{p^{t} p}{\left(q^{t} q\right)^{2}} q+2 \frac{q^{t} p}{q^{t} q} p-2\left(\frac{q^{t} p}{q^{t} q}\right)^{2} q .
\end{aligned}
$$


Equations for $\dot{\phi}$ and $\dot{\phi}$ follow immediately:

$$
\begin{aligned}
\dot{\phi} & =q^{t} \dot{q}=q^{t} p-2 q^{t} p+\frac{q^{t} q-1}{q^{t} q} q^{t} p=-\psi+\frac{\psi \phi}{\phi+1 / 2}, \\
\dot{\psi} & =q^{t} \dot{p}+p^{t} \dot{q}=-\frac{p^{t} p}{q^{t} q}+p^{t} p-2 \frac{\left(q^{t} p\right)^{2}}{q^{t} q}+p^{t} p \frac{q^{t} q-1}{q^{t} q} \\
& =2 p^{t} p \frac{\phi}{\phi+1 / 2}-\frac{\psi^{2}}{\phi+1 / 2} .
\end{aligned}
$$

The term $p^{t} p$ is a nuisance. If we treat it as a time-dependent coefficient, linearizing at $\phi=\psi=0$, we get

$$
\dot{\phi}=-\psi, \quad \dot{\psi}=4 p^{t} p \phi,
$$

which makes the origin a center; this agrees with the numerical experiment shown in Figure 3.

By contrast, if we had only made use of constraints on $q$ in formulating the system, we would have had, after following the above analysis and linearizing,

$$
\dot{\phi}=\psi, \quad \dot{\psi}=4 p^{t} p \phi,
$$

meaning that the origin has become a saddle point; this is exactly the situation we would expect from viewing Figure 4.

Although the general nonlinear case can be quite complicated, some generalization of the comparative analysis for linear systems of the first part of this section is possible via linearization of nonlinear constraints if we bear in mind that a potential energy function always has a positive definite Hessian at least in the neighborhood of a stable equilibrium [7]. On the other hand, all we can conclude from the stability of the linearized system is the absence of an exponential instability in the nonlinear system [2].

3.5. Weakly Hamiltonian underlying ODE. Dirac's process requires the differentiation of the constraint multipliers $\mu$ and $\eta$; since $\mu$ and $\eta$ depend on second derivatives of $g$ and first derivatives of $H$, construction of a Hamiltonian underlying ODE along the lines of Dirac's theory in general requires third derivatives of $g$ and second derivatives of $H$. However, along the constraint manifold $\mathscr{M}$, which is an invariant under the flow of (16)-(17), the terms multiplying the partial derivatives of $\mu$ and $\eta$ vanish, and we are left with a simplified system:

$$
\begin{aligned}
& \dot{q}=\nabla_{p} H+G^{t} \eta, \\
& \dot{p}=-\nabla_{q} H-G^{t} \mu-(G p)_{q}^{t} \eta .
\end{aligned}
$$

This system (referred to as the "Weakly Hamiltonian Dirac formulation") behaves like a Hamiltonian system for initial values chosen on the constraint manifold; in fact, any underlying ODE is a Hamiltonian system along the constraint manifold. But under numerical discretization we cannot in general expect the constraints to be maintained exactly, so that a canonical ODE discretization scheme applied to (30)-(31) would not result in a canonical step-to-step map. On the other hand, (30)-(31) requires only the computation of second derivatives of $g$ and first derivatives of $H$; hence it may be much more easily computed for certain problems. This formulation has been treated in the literature 
in connection with Lagrangian formulations of the equations of motion (see, e.g., [9]). The stability of the constraints is well understood for the weakly Hamiltonian formulations (30)-(31) and the standard underlying ODE (see, e.g., [14]). For linear constraints, (30)-(31) leads to

$$
\dot{q}=(I-\mathscr{H}) p, \quad \dot{p}=-(I-\mathscr{H}) \nabla F,
$$

and hence, in the previously used notation,

$$
\dot{r}=0, \quad \dot{s}=0
$$

for the projections $r=\mathscr{H} q, s=\mathscr{H} p$. For the standard underlying ODE, on the other hand, one obtains

$$
\dot{r}=s, \quad \dot{s}=0 .
$$

In other words, the constraints are weakly unstable. From this analysis, if one is not concerned with maintaining the symplectic structure, the formulation (30)(31) would seem to be the more desirable formulation. Since this formulation (as well as the standard underlying ODE) corresponds to the restriction of a Hamiltonian system to the constraint manifold, it is natural to ask whether we could not get away with solving one of these two simplified systems using a canonical integration method without too much damage to the energy. Numerical experiments with, respectively, the standard underlying ODE and (30)-(31) formulations are summarized in Figures 5 and 6 . We used a stepsize of $h=.1$ and solved the equations to tolerance $10^{-6}$.
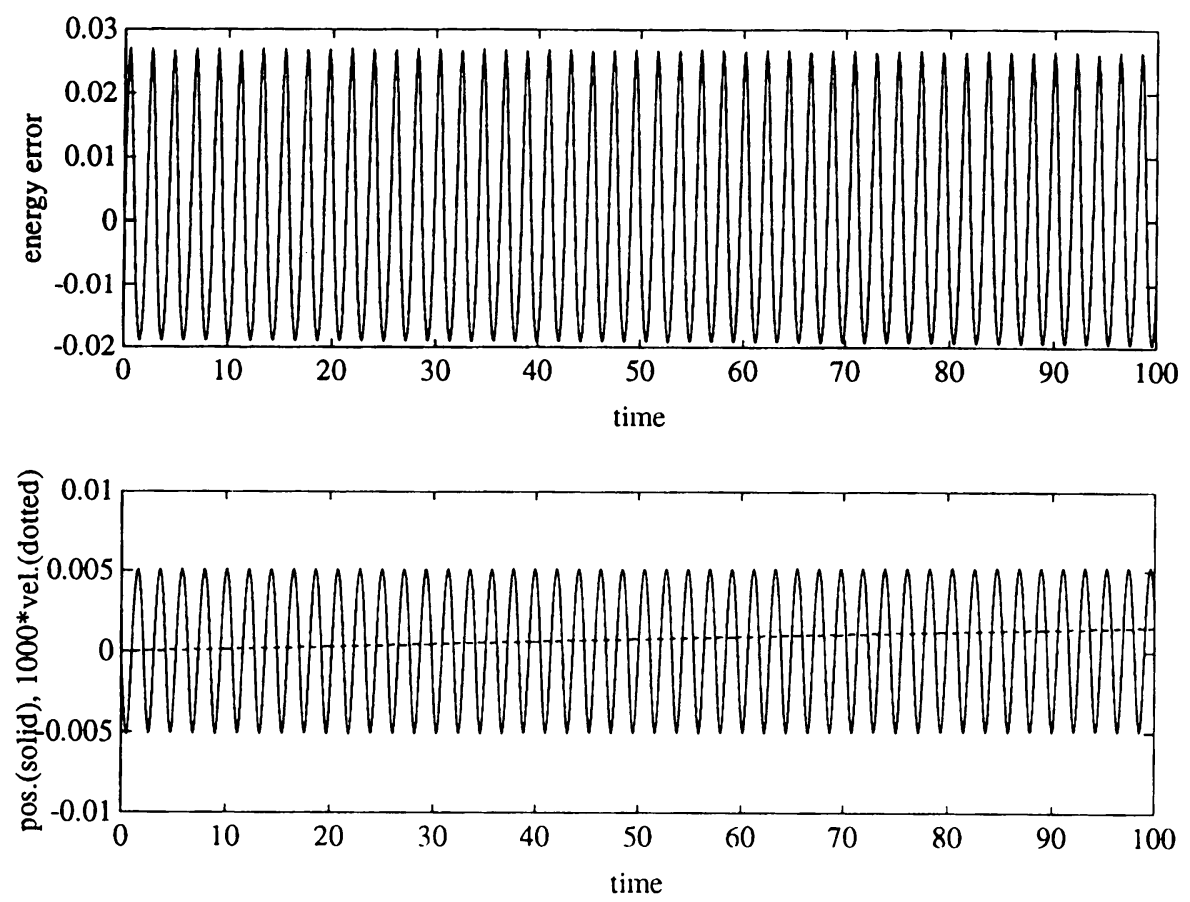

FIGURE 5. Standard underling ODE formulation of the pendulum: energy and constraint residuals 

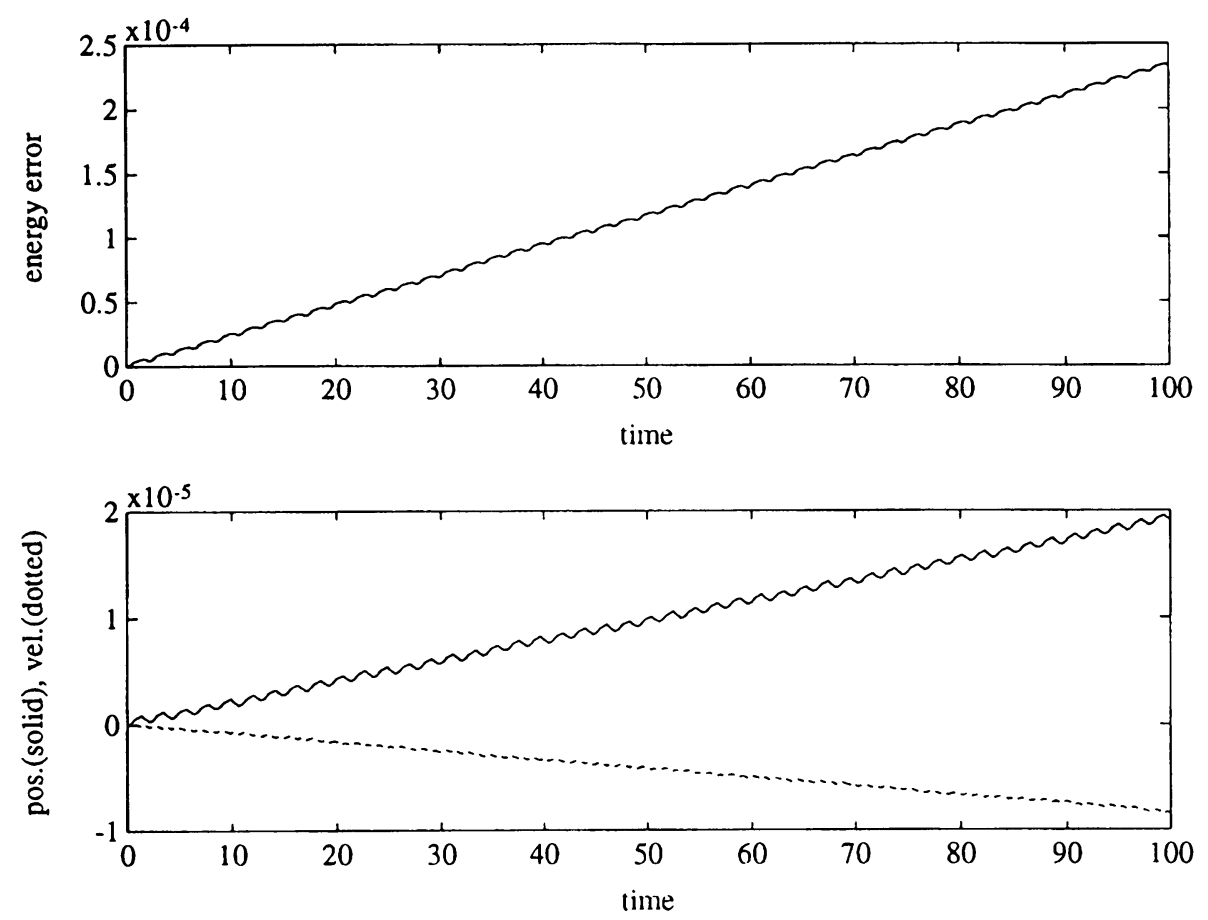

FIGURE 6. The weakly Hamiltonian Dirac formulation for the pendulum

These experiments seem to indicate that direct integration of the weakly Hamiltonian formulations with a canonical integrator may offer a practical, although nonsymplectic, alternative to the true Hamiltonian formulation, even on relatively long intervals. Note that the energy conservation observed in Figure 6 is far better than that observed in Figure 3, and somewhat better than that of Figure 5. It turns out that this is exceptionally good behavior owing to the combination of having a single quadratic constraint and using the implicit midpoint method, since the original Hamiltonian $H$ is a first integral of the reformulation in this case. This topic will be addressed in a future article.

\section{ACKNOWLEDGMENT}

We would like to thank Linda Petzold for pointing us to this problem, and Peter Deuflhard, Jacek Szmigielski, and the referee for their useful comments. We are especially grateful to the referee for assistance with $\S 2$.

\section{BIBLIOGRAPHY}

1. R. Abraham and J. E. Marsden, Foundations of mechanics, 2nd ed., Benjamin, Reading, MA, 1978.

2. V. I. Arnold, Mathematical methods of classical mechanics, Graduate Texts in Math., Vol. 60, Springer-Verlag, New York, 1975.

3. U. M. Ascher and L. R. Petzold, Stability of computational methods for constrained dynamics systems, SIAM J. Sci. Statist. Comput. 14 (1993), 95-120.

4. J. Baumgarte, Stabilization of constraints and integrals of motion in dynamical systems, Comp. Methods Appl. Mech. Engrg. 1 (1976), 1-16. 
5. K. E. Brenan, S. L. Campbell, and L. R. Petzold, Numerical solution of initial value problems in differential-algebraic equations, North-Holland, Amsterdam, 1989.

6. G. J. Cooper, Stability of Runge-Kutta methods for trajectory problems, IMA J. Numer. Anal. 7 (1987), 1-13.

7. R. Courant and D. Hilbert, Methods of mathematical physics, Wiley, New York, 1953.

8. P. A. M. Dirac, Lectures on quantum mechanics, Belfer Graduate School Monographs, no. 3, Yeshiva University, 1964.

9. E. Eich, C. Führer, B. Leimkuhler, and S. Reich, Stabilization and projection methods for multibody dynamics, Report A281, Helsinki Univ. of Technology, Helsinki, 1990.

10. L. Jay, Symplectic partitioned Runge-Kutta methods for constrained Hamiltonian systems, Technical Report, Université de Genève, 1993.

11. B. Leimkuhler and S. Reich, Numerical methods for constrained Hamiltonian systems, Technical Report, Konrad Zuse Center, Berlin, 1992.

12. B. Leimkuhler and R. D. Skeel, Symplectic numerical integrators for constrained molecular dynamics, Technical Report, Dept. of Math., University of Kansas, Lawrence KS, 1992.

13. N. H. McClamroch and A. M. Bloch, Control of constrained Hamiltonian systems and applications to control of constrained robots, Dynamical Systems Approaches to Nonlinear Problems in Systems and Circuits (Fathi M. A. Salam and Mark L. Levi, eds.), SIAM, Philadelphia, PA, 1988, pp. 344-403.

14. T. Mrziglod, Zur Theorie und numerischen Realisierung von Lösungsmethoden bei Differentialgleichungen mit angekoppelten algebraischen Gleichungen, Diplomarbeit, Math. Inst., Univ. zu Köln, 1987.

15. J. M. Sanz-Serna, Symplectic integrators for Hamiltonian problems: an overview, Acta $\mathrm{Nu}$ mer. 1 (1991).

16. P. Olver, Applications of Lie groups to differential equations, Springer-Verlag, Berlin and New York, 1986.

17. F. Potra and W. Rheinboldt, On the numerical solution of the Euler-Lagrange equations, NATO Advanced Research Workshop on Real-Time Integration Methods for Mechanical System Simulation (R. Deyo and E. Haug, eds.), Springer-Verlag, Berlin and New York, 1990.

18. S. Reich, Symplectic integration of constrained Hamiltonian systems by Runge-Kutta methods, Technical Report 93-13, Dept. of Comput. Sci., University of British Columbia, 1993.

19. W. Rheinboldt, Numerical analysis of parametrized nonlinear equations, Univ. Ark. Lecture Notes in Math. Sci., vol. 7, Wiley, New York, 1986.

20. J. M. Sanz-Serna, Runge-Kutta schemes for Hamiltonian systems, BIT 28 (1988), 877-883.

Department of Mathematics, University of Kansas, Lawrence, Kansas 66045-2142

E-mail address: leimkuhl@math. ukans .edu

Institute of Applied Mathematics and Statistics, Berlin O-1086, Germany

Current address: University of British Columbia, Vancouver, British Columbia, Canada V6T 1 Z2 